\title{
Clinical Applications of 3D T2-weighted MRI in Pelvic Imaging
}

\section{Authors:}

Kelvin K Lim, MBBS FRANZCR

Geertje Noe, MD FRANZCR

Emma Hornsey, BAppSc

Ruth P Lim, MBBS MMed MS FRANZCR

\section{Corresponding Author:}

Kelvin K Lim

Austin Health

Department of Radiology

145 Studley Road

P.O. Box 5555

Heidelberg

Victoria

Australia, 3084

Telephone: +61394965431

Fax: +6139459 2817

Email: kelv_lim@yahoo.com

\section{Institution:}

Austin Hospital, Department of Radiology, Heidelberg, Victoria 3084, Australia (department affiliated with University of Melbourne, Melbourne Medical School)

\section{Grant Support:}

None.

Presented as an e-poster and static poster at the Royal Australian and New Zealand College of Radiologist (RANZCR) Annual Scientific Meeting 2013, Auckland, New Zealand. 
Clinical Applications of 3D T2-weighted MRI in Pelvic Imaging

\section{$\underline{\text { Abstract }}$}

Objective: The purpose of this article is to illustrate clinical applications of 3D T2 weighted MRI in pelvic imaging. We review technical considerations of 3D T2 weighted MRI, with clinical examples.

Conclusion: 3D T2 weighted MRI has been increasingly utilized for pelvic applications, including imaging of rectal cancer, prostate cancer, anorectal fistulas and the female pelvis. This relatively rapid technique offers good softtissue contrast of the pelvic organs, with potential for more widespread clinical use.

\section{Introduction}

T2-weighted sequences are essential in evaluation of pelvic pathology, providing good soft tissue contrast of the pelvic organs [1]. Standard twodimensional (2D) turbo-spin-echo-based T2- weighted imaging (2D T2WI) performed in multiple planes traditionally plays a crucial role in clinical protocols for pelvic MR imaging. A relatively rapid 3D turbo spin-echo T2 technique (3D T2WI) has been described [2] which has recently been validated for specific pelvic applications by several centers.

The purpose of this review is to provide an overview of 3D T2WI for pelvic imaging. This will include a brief discussion of the basics of the sequence, its implementation for pelvic applications, and advantages and limitations of the technique when compared to conventional 2D T2WI. We demonstrate various applications in the context of illustrative clinical examples.

\section{Physics of 3D T2WI}

3D turbo spin echo (TSE) T2 weighted imaging has traditionally been limited by usable echo train length, specific absorption ration (SAR) and long acquisition times [3].Consequently, multi-planar 2D T2WI, typically in 3 orthogonal planes, has been the gold standard for T2 weighted pelvic imaging. Sampling Perfection with Application optimized Contrasts using different flip angle Evolutions (SPACE) is a 3D TSE sequence that overcomes some of the problems that have limited standard 3D TSE acquisitions.

SPACE employs non-selective, refocusing echo trains with the option for constant or variable flip angle refocusing pulses. Reducing and varying the flip angles (FA) minimizes interecho spacing and allows the length of the usable echo train to be significantly increased with benefits to SAR. In constant refocusing FA mode, flip angles are varied briefly at the start of the echo train then followed by constant flip angles for the remainder of the echo 
train. In the variable refocusing FA mode, higher amplitude FAs are applied at the beginning of the echo train, with subsequent FAs slowly decreased and varied over time [2]. The resultant "pseudo" steady state produced in both modes decays slowly maintaining signal to noise ratio (SNR) throughout the acquisition. Tissue contrast comparable to traditional T2 weighted 2D spin echo sequences is achieved by optimizing flip angles to maximize relaxation effects of tissues of interest and a complex mixture of spin and stimulated echoes allows a contrast equivalent TE to be achieved.

Combining low SAR, long echo trains with parallel imaging and partial Fourier acquisition allow high resolution $3 \mathrm{D}$ T2WI to be achieved within reasonable acquisition times. For example, a $1 \times 1 \times 1 \mathrm{~mm}$ isotropic voxel T2 weighted SPACE volume of the whole pelvis can be achieved in less than eight minutes. Comparatively, single plane 2D TSE imaging of the pelvis with $3 \mathrm{~mm}$ non-contiguous slices can be achieved in approximately 4 minutes.

A typical protocol for pelvic MRI would include a three plane localizer, coronal T2 SPACE of the whole pelvis, axial T1 whole pelvis, axial T2 fat saturated whole pelvis and axial DWI over the region of interest. At our institution, this protocol is used in rectal, cervical and prostate cancer staging. Imaging of endometriosis and anorectal fistulas would often include additional sequences such as coronal T1 fat saturated sequence and mid sagittal HASTE/ True FISP sequence in an endometriosis examination and 3-plane (sagittal, oblique axial, oblique coronal) T2 fat saturated BLADE sequences in a fistula study. An example of typical imaging parameters used for 3D constant refocusing radiofrequency ( $R F$ ) mode versus $2 \mathrm{D}$ T2WI pelvic imaging, in addition to parameters of the other sequences performed in a pelvic study, with a standard 6-channel body phased array receive coil at 1.5T is shown in Table 1.

\section{Implementation for pelvic imaging}

Implementation of 3D T2WI for abdominal and pelvic applications may include fat suppression, which is useful in improving contrast between fat and T2 hyperintense structures, for example, perianal fistulas. Similarly, intra-cavitary contrast, intra-cavitary coils and antispasmodics may aid image quality and interpretation for specific indications. Potential benefits of these should be weighed against potential disadvantages, including increased patient discomfort and decreased workflow efficiency.

\section{Fat suppression}

Fat suppression for 3D T2 weighted imaging can be achieved with a short tau inversion recovery (STIR) SPACE sequence. A non-selective magnetization preparation pulse, with an inversion time to null fat (160ms at $1.5 \mathrm{~T})$, is applied at the start of the sequence. Imaging times are comparable to T2 SPACE but resolution is sacrificed (for example, $1.3 \mathrm{~mm}^{3}$ isotropic voxels) to achieve reasonable imaging times and adequate SNR.

\section{Intracavitary contrast}

Ultrasound gel is used at most centers where intra-cavitary contrast is utilized [4]. Some institutions recommend against the use of endorectal contrast [5] 
altogether for rectal cancer staging while others advocate the use of endorectal contrast for rectal tumour staging in small, polypoid or previously treated tumours, but suggest it should be avoided in large and low rectal tumours [6].

Similarly, intra-vaginal contrast may be used, particularly in suspected deep pelvic endometriosis or assessment of cervical carcinoma [7-9].

\section{Intracavitary coils}

Intracavitary coils improve SNR in close proximity to the coil, enabling small volume, high spatial resolution imaging, and have been described for evaluation of cervical, prostate and rectal tumors [10,11]. Challenges of intracavitary coils include a limited field of view due to limited depth of signal reception, difficulty in correct positioning relative to the pathology, hyperintense signal, anatomic distortion of adjacent tissues and motion artifact from rectal peristalsis. Endorectal coils may be difficult to use in patients with stenosing rectal tumours and may be contra-indicated for example in patients who have undergone recent surgery or radiation therapy, anorectal fistula and inflammatory bowel disease involving the rectum [12, 13]. Cost and time of performing an MRI is increased when an endorectal coil is used [14].

The 2012 European Society of Urogenital Radiology (ESUR) prostate MR guidelines still recommend a combination of endorectal and pelvic phased array coils for prostate cancer staging, but state cost and decreased patient tolerance as drawbacks, and cite literature demonstrating good results for multiparametric MRI tumour detection without endorectal coils[15].

Imaging at $3 \mathrm{~T}$ has a theoretical potential to improve signal to noise ratio twofold enabling improvements in spatial resolution $[14,16]$. Most drawbacks at 3T described in the ESUR guidelines are currently being overcome [15]. Studies comparing endorectal coils versus external phased array coils at 3T suggest that image quality between the two techniques is at least comparable $[14,17,18]$.

\section{Antispasmodics}

Antispasmodics are useful to reduce bowel peristalsis and related motion artifact, particularly important for 3D T2WI. They are recommended for prostate imaging to reduce bowel motion artifacts [15]. For rectal cancer staging, they are considered to be useful but not mandatory for improving image quality [5]. They may also aid in evaluation of bowel involvement in deep endometriosis, and in cervical and endometrial cancer. Commonly used agents are glucagon and hyoscine butylbromide (Buscopan; Boehringer Ingelheim, Ingelheim, Germany).

\section{Advantages of 3D versus 2D T2 Imaging}


Pelvic MRI often requires imaging in non-standard planes. For example, imaging along the long axis of the uterus is essential to evaluate uterine congenital anomalies [19]. 3D T2WI allows interactive reconstruction in any orientation with isotropic resolution, which can be obtained off-line after the examination, improving examination efficiency and offering a useful problem solving tool [20]. In contrast, 2D T2WI does not allow retrospective post processing if coverage or imaging planes have been incorrectly prescribed.

Time savings using 3D T2WI of up to $50-60 \%$ have been reported. For example, prostate cancer staging MRI performed in a study by Rosenkrantz et al. demonstrated significant time savings, with acquisition time of 4 minutes, in contrast to approximately 11 minutes for 3-plane 2D T2WI [21]. Similar time savings have been documented in rectal and endometrial cancer assessment [22, 23].

Wang et al. has reported use of localisation tool on 2D T2-weighted images of the prostate in multiple planes improves accuracy for detection of extracapsular extension \& seminal vesicle invasion [24]. Localisation is theoretically more accurate when used with multiplanar imaging reconstructed from a single 3D data set, as there is a risk of motion-related misregistration between separate 2D sequences.

Another benefit is relatively large field of view imaging, with whole pelvis coverage feasible in shorter scan time than multiplanar 2D T2WI with comparable spatial resolution, without interslice gaps. In 2D T2WI, slice gaps to prevent cross talk preclude true volumetric coverage [25].

\section{Limitations of 3D versus 2D T2 Imaging}

\section{T2 contrast}

3D T2WI can theoretically result in reduced T2 contrast, which might hinder lesion detection for oncologic applications and render free fluid of similar signal intensity to fat. This is thought to be due to use of repeated refocusing pulses less than $180^{\circ}$, resulting in greater $\mathrm{T} 1$ dependence [21, 26, 27], however use of a restore pulse [28] can mitigate this problem. Decreased fluid conspicuity suggests a single conventional $2 \mathrm{D}$ sequence alongside the $3 \mathrm{D}$ sequence may be helpful.

However, Rosenkrantz et al. reported preserved contrast to noise ratio with 3D T2WI for prostate imaging, despite theoretical alteration of T2 contrast compared with standard 2D T2 MRI [21, 27]. Although 2D had higher signal to noise ratio (SNR) in this study, image quality was the same subjectively between 2D and 3D.

\section{Spatial Resolution}

Although 3D T2WI offers potential for isotropic spatial resolution, Fütterer et al reported that lower in-plane resolution of $1 \mathrm{~mm} \times 1 \mathrm{~mm}$ in 3D T2WI had inferior sensitivity, specificity and accuracy to $0.39 \mathrm{~mm} \times 0.39 \mathrm{~mm} 2 \mathrm{D}$ T2WI in-plane resolution for identifying T3 rectal cancer [13]. More motion related artefacts were also reported for 3D T2WI, likely related to longer acquisition time and bowel movement [13]. 


\section{B1 Inhomogeneity}

B1 inhomogeneity causes substantial variations of the flip angle across the FOV with 3D T2 TSE, particularly at 3T, which can cause significant variations in SNR and degrade image contrast and diagnostic quality, as reported for the sequence applied to non-contrast MRA [27, 29-31].

A technique to minimize artifact from B1 heterogeneity is use of a radiofrequency cushion [32-34]. Ongoing technologic improvements may eventually further reduce negative effects of this artifact on 3D T2 MRI by radiofrequency pulse optimization, including parallel transmission [35-37].

\section{SAR Limits/ RF deposition}

3D T2WI has potential benefit at 3T because it imparts lower radiofrequency deposition for lower SAR [26].

\section{Clinical Applications}

\section{$\underline{\text { Rectal Cancer }}$}

Colorectal cancer is a leading cause of mortality in the developed world. Given the prognosis of rectal cancer is closely related to the stage at diagnosis and involvement of the circumferential resection margin, preoperative staging, including assessment of local and systemic spread is crucial for optimal patient management [13]. Imaging features of rectal cancer include focal mass or mural thickening, with ancillary features of pericolonic infiltration and local lymphadenopathy depending on stage of disease.

MRI has been increasingly used for preoperative assessment and current literature has shown MRI to be effective in evaluation of local tumour status. $\mathrm{Kim}$ at al. showed that whilst tumor conspicuity was significantly higher for 2D TSE [23], there was no significant difference in overall image quality for artifacts, nor T staging (64.5\% for $2 \mathrm{D}$ versus $62 \%$ for $3 \mathrm{D}$ ) or $\mathrm{N}$ staging (2D $56 \%$ versus 3D 60\%). Examples are shown in Figures 1 to 3.

Whilst Beets-Tan et al., in a concensus statement from the European Society of Gastrointestinal and Abdominal Radiology (ESGAR) meeting, does not recommend the use of 3D T2 MRI in rectal cancer staging, in favour of multiplanar 2D T2 sequences, this did not appear to be secondary to any deficiencies in the 3D T2 technique, but rather a lack of current evidence to support the accuracy of this technique [38].Thus, further validation is required before more widespread clinical utility.

Futterer et al [13] states that the combination of the 3D SPACE and axial 2D T2-weighted MR images allowed accurate local staging and assessment of pelvic structures for surgical planning, however does not recommend the 3D SPACE as the sole T2 sequence given it's inferior differentiation of the rectal wall layers and lower T2 contrast. Given the above evidence, protocols for pelvic imaging therefore varies widely around world and is heavily dependent on local experience and expertise, with some institution performing 3D 
SPACE supplemented by a single plane 2D T2 TSE sequence (usually axial), whilst others perform the 3D SPACE as their exclusive T2 sequence, with the 2D T2 TSE available for trouble-shooting as necessary on a case-by-case basis.

\section{Prostate Cancer}

Prostate cancer is the most common cancer among men in the United Kingdom and United States [39]. In current practice, clinicians rely on digital rectal examination (DRE), serum prostate specific antigen (PSA) and transrectal ultrasound (TRUS) as diagnostic tools. Advances in prostate MRI has aided in detection and characterisation of prostate cancer using a multiparametric approach [40]. Prostate cancer in the peripheral zone typically demonstrate low T2 signal with corresponding low signal on ADC [41]. Additional features include extension of abnormal low signal tissue beyond the prostatic 'capsule' (ie: extracapsular extension) and invasion of the seminal vesicles.

Rosenkrantz et al. reported equivalent performance of 3D T2WI for tumor detection \& staging, with equivalent image quality and better tissue contrast for peripheral zone tumors compared to 2D TSE [21]. There was no significant difference in accuracy (2D 69.3\% versus 3D 69.7\%), sensitivity and specificity for prostate cancer detection on a per-sextant basis. There was no significant difference between the techniques for extracapsular extension evaluation (respective accuracies of $73.7 \%$ and $68.4 \%$, with $p=0.25-1$ ). An example is shown in Figure 4.

\section{Anorectal Fistulas}

Characteristic MRI signal of anorectal fistulas depends on it's activity and chronicity. More active or recent fistulas show low T1 and high T2 signal with intense enhancement of the fistula walls owing to the presence of granulation tissue. More chronic fistulas demonstrate low T1 and T2 signal without contrast enhancement.

Involvement of the anal sphincter complex by anorectal fistula can be accurately defined with MRI, vital for management planning and prediction of post-operative faecal incontinence. Hidden areas of sepsis and secondary extension that are occult to examination can be clearly delineated. 3D T2WI enables continuous coverage and depiction of fistulous tracts can be reconstructed in any plane [1] (Fig 5).

\section{Female Pelvis}

Due to organ mobility, female pelvic anatomy is very variable with variation in optimal imaging planes between individuals, placing limitations on coverage and orientation with 2D T2WI. High potentially isotropic spatial resolution of $3 \mathrm{D}$ imaging is another benefit for assessing small or subtle abnormalities.

\section{Endometriosis}

Endometriosis is a relatively common gynaecological disorder affecting 10$15 \%$ of women of reproductive age, with major impact on everyday life and 
fertility [42]. Deep (pelvic) infiltrating endometriosis (DIE) is particularly debilitating.

Endometriomas typically display high T1 signal contents, with variable T2 signal. A useful sign is the inhomogenous low signal of the cyst contents on T2 weighted sequences, a feature called 'T2 shading'. Endometriotic plaques demonstrate variable signal and their imaging characteristics are therefore neither sensitive nor specific [43]

3D T2WI has reportedly comparable or possibly superior diagnostic accuracy to conventional 2D T2WI for DIE assessment $[42,44]$. Identification of bladder and rectosigmoid involvement (Fig 6) has important implications for surgical planning and treatment as it may require more extensive surgery [43-46].

\section{Gynaecological malignancies/neoplasms}

Endometrial carcinoma is the most common and cervical carcinoma the third most common form of gynaecological cancer [22, 47]. Although not included in FIGO staging recommendations, largely due to limited global availability, MRI use has grown over the past two decades. It is widely accepted as a useful tool for pre-operative staging [47-49] and treatment planning, particularly whether surgery alone is appropriate (cervical tumors staged IIA or below), or whether chemo-radiation is indicated (Fig 7). Cervical cancer is usually $\mathrm{T} 1$ isointense and T2 hyperintense when compared to adjacent normal cervical stroma. Contrast enhancement is variable and therefore not useful in assessment [50]. Additional information to be gleaned from MRI include assessment of the parametrial tissues, pelvic side walls, ascites and uterine size $[50,51]$.

Staging of endometrial cancer is primarily surgical, but MRI has also been recognized as the best imaging tool for assessing myometrial invasion with important prognostic implications (Fig 8). It is useful in determining appropriate candidates for surgery, and surgical extent/ approach in eligible patients. Endometrial cancer usually demonstrate iso to low signal relative to normal endometrium on T1. It can demonstrate high T2 signal, but this is typically rather heterogenous. The tumour often enhances less avidly compared to myometrium and cervix in the equilibrium phase [50].

The 2009 revised FIGO criteria has had a significant impact for endometrial cancer staging, with staging simplification for endometrial cancer, potentially improving diagnostic accuracy of MRI [47]. An audit was recently performed in the UK, which looked into the accuracy of MRI staging of endometrial cancer and this audit reported an accuracy of $82 \%$ for myometrial invasion, $90 \%$ for cervical extension and pelvic nodal involvement of $94 \%$ [52].

\section{Congenital uterine anomalies}

Correct diagnosis of uterine anomalies (Müllerian duct anomalies) has implications for fertility and the ability to carry a pregnancy to full term. 3D T2 $\mathrm{MRI}$ is helpful for evaluating such a mobile organ [20,53], and large coverage enables simultaneous assessment of renal abnormalities, which co-exist in $30 \%$. The most common anomaly is septate uterus (Fig 9), which accounts for approximately $55 \%$ of cases [54]. It has a strong association with 
reproductive failure and is surgically correctable. It can be hard to differentiate from bicornuate uterus or uterus didelphys at ultrasound or hysterosalpingography but is easily identified at MRI [55].

\section{Future directions}

High field strength MR scanners (3T) are proliferating in clinical practice worldwide. The increased signal-to-noise ratio inherent to 3T compared to 1.5T facilitates increased spatial resolution imaging, which may translate into improved diagnostic performance particularly with regard to oncologic applications, although further comparative studies with 1.5T are required [13]. Clinical validation may eventually lead to replacement of multiplanar 2D TSE T2WI with a single volumetric acquisition with benefits for workflow efficiency.

3D T2 weighted MR has also shown promise in non-pelvic utilization. It has been used in 3D MR cholangiopancreatography at 3T, which has been shown to improve bile duct visualization and reduce artifacts [56]. Meindl et al. demonstrated 3D T2WI of the cervical spine afforded superior anatomical delineation and significantly fewer image artifacts of reconstructed 3D T2WI compared to axial 2D T2-weighted images [57].

\section{Conclusion}

T2-weighted sequences are essential in evaluation of pelvic pathology, providing good soft-tissue contrast of the pelvic organs. Standard 2D T2WI performed in multiple planes traditionally play a crucial role in clinical pelvic $\mathrm{MRI}$. As illustrated in this review, the relatively rapid 3D T2WI shows great promise in rectal cancer, prostate cancer, anorectal fistula and gynaecologic imaging. Future research should focus on its validation against 2D T2WI with a view to more widespread clinical use.

\section{References}

1. de Miguel Criado, J., et al., MR imaging evaluation of perianal fistulas: spectrum of imaging features. Radiographics, 2012. 32(1): p. 175-94.

2. Mugler, J., H. Meyer, and B. Kiefer. Practical implementation of optimized tissue-specific prescribed signal evolutions for improved turbo-spin-echo imaging [abstr]. in In: Proceedings of the Eleventh Meeting of the International Society for Magnetic Resonance in Medicine. Berkeley, Calif: International Society for Magnetic Resonance in Medicine, 2003. 2003. Toronto.

3. Scott, K. and S. Schmeets, SPACE: An Innovative Solution to Rapid Low SAR T2-weighted Contrast in 3D Spin Echo Imaging, in Magnetom Flash. 2005. p. 92-95. 
4. Kim, S.H., et al., Sonography transmission gel as endorectal contrast agent for tumor visualization in rectal cancer. AJR Am J Roentgenol, 2008. 191(1): p. 186-9.

5. Taylor, F.G., et al., A systematic approach to the interpretation of preoperative staging MRI for rectal cancer. AJR Am J Roentgenol, 2008. 191(6): p. 1827-35.

6. Kaur, $\mathrm{H}$., et al., MR imaging for preoperative evaluation of primary rectal cancer: practical considerations. Radiographics, 2012. 32(2): $p$. 389-409.

7. Akata, D., et al., Efficacy of transvaginal contrast-enhanced MRI in the early staging of cervical carcinoma. Eur Radiol, 2005. 15(8): p. 172733.

8. Marcal, L., et al., Deep pelvic endometriosis: MR imaging. Abdom Imaging, 2010. 35(6): p. 708-15.

9. Novellas, S., et al., [Anatomy of the female pelvis on MRI: value of intravaginal contrast]. J Radiol, 2009. 90(7-8 Pt 1): p. 819-24.

10. Huch Boni, R.A., et al., Contrast-enhanced endorectal coil MRI in local staging of prostate carcinoma. J Comput Assist Tomogr, 1995. 19(2): p. 232-7.

11. Vogl, T.J., et al., Accuracy of staging rectal tumors with contrastenhanced transrectal MR imaging. AJR Am J Roentgenol, 1997. 168(6): p. 1427-34.

12. Grand, D.J., W.W. Mayo-Smith, and C.A. Woodfield, Practical Body MRI: Protocols, Applications and Image Interpretation. 2012: Cambridge University Press.

13. Futterer, J.J., et al., Preoperative $3 T M R$ imaging of rectal cancer: local staging accuracy using a two-dimensional and three-dimensional T2weighted turbo spin echo sequence. Eur J Radiol, 2008. 65(1): p. 6671.

14. Sosna, J., et al., MR imaging of the prostate at 3 Tesla: comparison of an external phased-array coil to imaging with an endorectal coil at 1.5 Tesla. Acad Radiol, 2004. 11(8): p. 857-62.

15. Barentsz, J.O., et al., ESUR prostate MR guidelines 2012. Eur Radiol, 2012. 22(4): p. 746-57.

16. EM, H., Magnetic resonance imaging:Physical principles and sequence design. 1st ed. 1999, New York, NY: Wiley and Sons.

17. Kim, B.S., et al., Comparison of pelvic phased-array versus endorectal coil magnetic resonance imaging at 3 Tesla for local staging of prostate cancer. Yonsei Med J, 2012. 53(3): p. 550-6. 
18. Torricelli, P., et al., Comparative evaluation between external phased array coil at $3 \mathrm{~T}$ and endorectal coil at $1.5 \mathrm{~T}$ : preliminary results. $\mathrm{J}$ Comput Assist Tomogr, 2006. 30(3): p. 355-61.

19. Patel, V.H. and S. Somers, MR imaging of the female pelvis: current perspectives and review of genital tract congenital anomalies, and benign and malignant diseases. Crit Rev Diagn Imaging, 1997. 38(5): p. 417-99.

20. Proscia, N., et al., MRI of the pelvis in women: $3 D$ versus $2 D$ T2weighted technique. AJR Am J Roentgenol, 2010. 195(1): p. 254-9.

21. Rosenkrantz, A.B., et al., Prostate cancer: Comparison of 3D T2weighted with conventional 2D T2-weighted imaging for image quality and tumor detection. AJR Am J Roentgenol, 2010. 194(2): p. 446-52.

22. Hori, M., et al., Uterine tumors: comparison of 3D versus 2D T2weighted turbo spin-echo MR imaging at 3.0 T--initial experience. Radiology, 2011. 258(1): p. 154-63.

23. Kim, H., et al., Rectal cancer: comparison of accuracy of local-regional staging with two- and three-dimensional preoperative 3-T MR imaging. Radiology, 2010. 254(2): p. 485-92.

24. Wang, L., et al., Incremental value of multiplanar cross-referencing for prostate cancer staging with endorectal MRI. AJR Am J Roentgenol, 2007. 188(1): p. 99-104.

25. Brown, M. and R. Semelka, MRI: Basic Principles and Applications. 4th ed. 2011, USA: Wiley-Blackwell. 264.

26. Hecht, E.M., et al., Preliminary clinical experience at $3 T$ with a 3D T2weighted sequence compared with multiplanar 2D for evaluation of the female pelvis. AJR Am J Roentgenol, 2011. 197(2): p. W346-52.

27. Lichy, M.P., et al., Magnetic resonance imaging of the body trunk using a single-slab, 3-dimensional, T2-weighted turbo-spin-echo sequence with high sampling efficiency (SPACE) for high spatial resolution imaging: initial clinical experiences. Invest Radiol, 2005. 40(12): p. 75460.

28. Reimer, P., P.M. Parizel, and F.-A. Stichnoth, Clinical MR Imaging: A Practical Approach. 2 ed, ed. P. Reimer. 2006: Springer. 597.

29. Lim, R.P., et al., Imaging the female pelvis at 3.0 T. Top Magn Reson Imaging, 2006. 17(6): p. 427-43.

30. Rosenkrantz, A.B., et al., Liver MRI at $3 T$ using a respiratory-triggered time-efficient 3D T2-weighted technique: impact on artifacts and image quality. AJR Am J Roentgenol, 2010. 194(3): p. 634-41. 
31. Storey, P., et al. B1 inhomogeneity in the thigh at $3 T$ and implications for peripheral vascular imaging. in Proceedings of the 17th Annual Meeting of ISMRM, Honolulu, Hawaii, USA. 2009.

32. Haystead, C.M., B.M. Dale, and E.M. Merkle, N/2 ghosting artifacts: elimination at 3.0-T MR cholangiography with SPACE pulse sequence. Radiology, 2008. 246(2): p. 589-95.

33. Franklin, K.M., B.M. Dale, and E.M. Merkle, Improvement in B1inhomogeneity artifacts in the abdomen at $3 T$ MR imaging using a radiofrequency cushion. J Magn Reson Imaging, 2008. 27(6): p. 14437.

34. Kataoka, M., et al., MR imaging of the female pelvis at 3 Tesla: evaluation of image homogeneity using different dielectric pads. J Magn Reson Imaging, 2007. 26(6): p. 1572-7.

35. Saekho, S., et al., Small tip angle three-dimensional tailored radiofrequency slab-select pulse for reduced B1 inhomogeneity at $3 T$. Magn Reson Med, 2005. 53(2): p. 479-84.

36. Setsompop, K., et al., Parallel RF transmission with eight channels at 3 Tesla. Magn Reson Med, 2006. 56(5): p. 1163-71.

37. Park, J., J.P. Mugler, 3rd, and T. Hughes, Reduction of B1 sensitivity in selective single-slab 3D turbo spin echo imaging with very long echo trains. Magn Reson Med, 2009. 62(4): p. 1060-6.

38. Beets-Tan, R.G., et al., Magnetic resonance imaging for the clinical management of rectal cancer patients: recommendations from the 2012 European Society of Gastrointestinal and Abdominal Radiology (ESGAR) consensus meeting. Eur Radiol, 2013. 23(9): p. 2522-31.

39. Jemal, A., et al., Cancer statistics, 2009. CA Cancer J Clin, 2009. 59(4): p. 225-49.

40. Dickinson, L., et al., Magnetic resonance imaging for the detection, localisation, and characterisation of prostate cancer: recommendations from a European consensus meeting. Eur Urol, 2011. 59(4): p. 477-94.

41. Rosenkrantz, A.B., et al., Prostate cancer: comparison of tumor visibility on trace diffusion-weighted images and the apparent diffusion coefficient map. AJR Am J Roentgenol, 2011. 196(1): p. 123-9.

42. Bazot, M., et al., Comparison of 3D and 2D FSE T2-weighted MRI in the diagnosis of deep pelvic endometriosis: preliminary results. Clin Radiol, 2013. 68(1): p. 47-54.

43. Woodward, P.J., R. Sohaey, and T.P. Mezzetti, Jr., Endometriosis: radiologic-pathologic correlation. Radiographics, 2001. 21(1): p. 193216; questionnaire 288-94. 
44. Giusti, S., et al., Anatomical localization of deep infiltrating endometriosis: 3D MRI reconstructions. Abdom Imaging, 2012. 37(6): p. $1110-21$.

45. Del Frate, C., et al., Deep retroperitoneal pelvic endometriosis: $M R$ imaging appearance with laparoscopic correlation. Radiographics, 2006. 26(6): p. 1705-18.

46. Hottat, N., et al., Endometriosis: Contribution of 3.0-T Pelvic MR Imaging in Preoperative Assessment_Initial Results. Radiology, 2009. 253(1): p. 126-134.

47. Beddy, P., et al., FIGO staging system for endometrial cancer: added benefits of MR imaging. Radiographics, 2012. 32(1): p. 241-54.

48. Koyama, T., K. Tamai, and K. Togashi, Staging of carcinoma of the uterine cervix and endometrium. Eur Radiol, 2007. 17(8): p. 2009-19.

49. Bhosale, P., et al., Role of magnetic resonance imaging as an adjunct to clinical staging in cervical carcinoma. J Comput Assist Tomogr, 2010. 34(6): p. 855-64.

50. Sala, E., et al., MRI of malignant neoplasms of the uterine corpus and cervix. AJR Am J Roentgenol, 2007. 188(6): p. 1577-87.

51. Sala, E., et al., The added role of MR imaging in treatment stratification of patients with gynecologic malignancies: what the radiologist needs to know. Radiology, 2013. 266(3): p. 717-40.

52. Duncan, K.A., et al., Staging cancer of the uterus: a national audit of MRI accuracy. Clin Radiol, 2012. 67(6): p. 523-30.

53. Agrawal, G., et al., Evaluation of uterine anomalies: 3D FRFSE cube versus standard 2D FRFSE. AJR Am J Roentgenol, 2009. 193(6): p. W558-62.

54. Behr, S.C., J.L. Courtier, and A. Qayyum, Imaging of Müllerian Duct Anomalies. RadioGraphics, 2012. 32(6): p. E233-E250.

55. Junqueira, B.L.P., et al., Müllerian Duct Anomalies and Mimics in Children and Adolescents: Correlative Intraoperative Assessment with Clinical Imaging1. Radiographics, 2009. 29(4): p. 1085-1103.

56. Arizono, S., et al., High spatial resolution 3D MR cholangiography with high sampling efficiency technique (SPACE): comparison of $3 T$ vs. 1.5T. Eur J Radiol, 2010. 73(1): p. 114-8.

57. Meindl, T., et al., Magnetic resonance imaging of the cervical spine: comparison of 2D T2-weighted turbo spin echo, 2D T2*weighted gradient-recalled echo and $3 D$ T2-weighted variable flip-angle turbo spin echo sequences. Eur Radiol, 2009. 19(3): p. 713-21. 
58. Griswold, M.A., et al., Generalized autocalibrating partially parallel acquisitions (GRAPPA). Magn Reson Med, 2002. 47(6): p. 1202-10.

Figures

Table 1

\begin{tabular}{|l|c|c|c|c|c|}
\hline & 3D & 2D T2 & 2D Axial & 2D Axial T2 & Axial DWI \\
& SPACE & TSE & T1 SE & FS BLADE & \\
\hline
\end{tabular}




\begin{tabular}{|c|c|c|c|c|c|}
\hline TE (ms) & 131 & 72 & 9.4 & 74 & 2200 \\
\hline TR (ms) & 1500 & 4000 & 682 & 4000 & 83 \\
\hline $\begin{array}{l}\text { REFOCUSING FLIP } \\
\text { Angle }\left({ }^{\circ}\right)\end{array}$ & 150 & 150 & 150 & 150 & $\begin{array}{c}\text { b values } \\
50 / 400 / 800\end{array}$ \\
\hline Echo train length & 85 & 14 & None & 20 & $\begin{array}{l}144 \mathrm{EPI} \\
\text { Factor }\end{array}$ \\
\hline $\begin{array}{l}\text { INTERECHO SPACING } \\
\text { (ms) }\end{array}$ & 4.38 & 12 & 9.4 & 6.7 & 0.85 \\
\hline BANDWIDTH & 449 & 161 & 150 & 260 & 1302 \\
\hline FOV (mm) & 400 & 200 & 320 & 320 & 250 \\
\hline MATRIX & $384 \times 384$ & $256 \times 320$ & $320 \times 320$ & $\begin{array}{c}123 \% \\
\text { BLADE x } \\
384\end{array}$ & $144 \times 192$ \\
\hline $\begin{array}{l}\text { SLICE THICKNESS } \\
(\mathrm{mm})\end{array}$ & 1.0 & 3.0 & 5 & 5 & 4 \\
\hline SLICE GAP (mm) & $\mathrm{N} / \mathrm{A}$ & 0.3 & 1.25 & 1.25 & 1.6 \\
\hline SLICE NUMBER & 176 & 23 & 45 & 45 & 15 \\
\hline $\begin{array}{l}\text { Parallel imaging } \\
\text { factor (GRAPPA)[58] }\end{array}$ & 3 & 2 & & 2 & 2 \\
\hline $\begin{array}{l}\text { PARTIAL FOURIER } \\
\text { (phase) }\end{array}$ & $6 / 8$ & None & None & None & $6 / 8$ \\
\hline $\begin{array}{l}\text { ACQUISITION TIME } \\
\text { (minutes) }\end{array}$ & 7.41 & 4.0 & 4.23 & 4.26 & 4.04 \\
\hline
\end{tabular}

Table 1 - Typical imaging parameters used for 3D constant refocusing radiofrequency (RF) mode versus $2 \mathrm{D}$ T2WI pelvic imaging with a standard 6channel body phased array receive coil at 1.5T Parameters for the other sequences typically performed in a pelvic examination are also included.

GRAPPA = generalized autocalibrating partially parallel acquisition 
Fig. 1-69 year old female patient with a rectosigmoid carcinoma. Oblique sagittal reformatted image demonstrate the rectosigmoid carcinoma with loss of the hypointense muscularis propria (inferior surface) with invasion of the uterine fundus (arrows), in keeping with a stage T4 tumour.

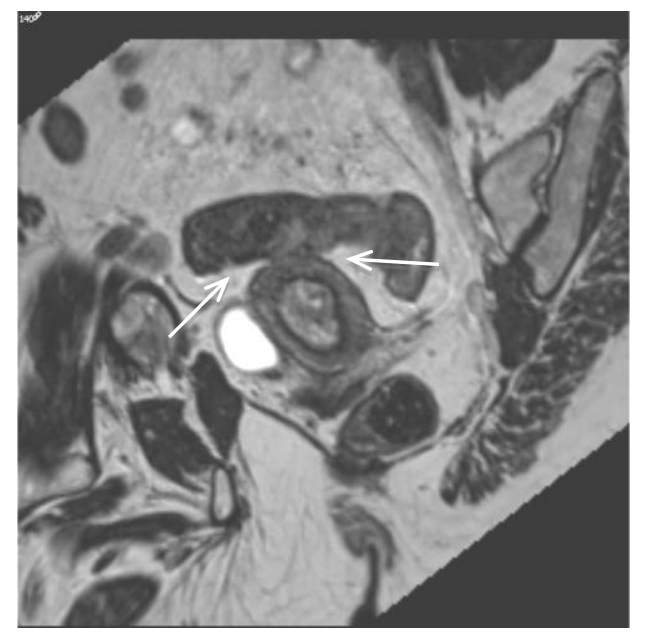

Fig. 2 - 59 year old male patient with a mid to low rectal carcinoma with near circumferential mural thickening.

A: Coronal reformat showing disruption of the left lateral wall muscularis propria and a peak of tumour tissue (arrow) extending into and along the lymphovascular bundle consistent with lymphovascular invasion.

B: Oblique axial image demonstrating 2 separate sites of lymphovascular invasion (arrows), not clearly delineated on standard axial or coronal images.
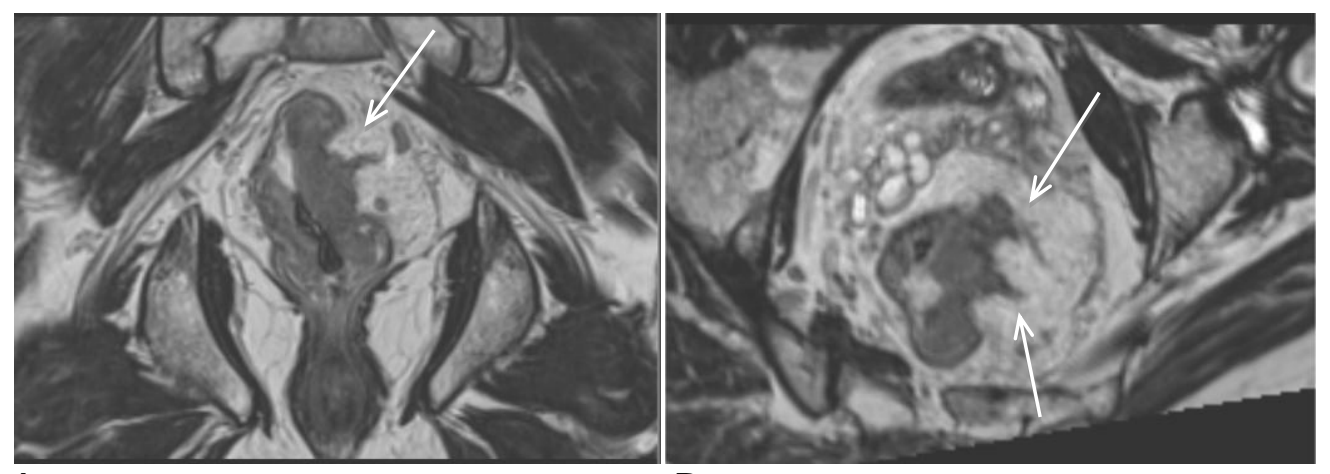

A

B 
Fig. 3-61 year old male patient with a rectal adenocarcinoma.

Coronal image demonstrating polypoid endorectal masses with a frondlike architecture, surrounded by high T2 signal material. This was confirmed to be a mucin-secreting rectal adenocarcimoma on histopathology. Note the intact muscularis propria (arrows) in keeping with a stage $\leq \mathrm{T} 2$ tumour.

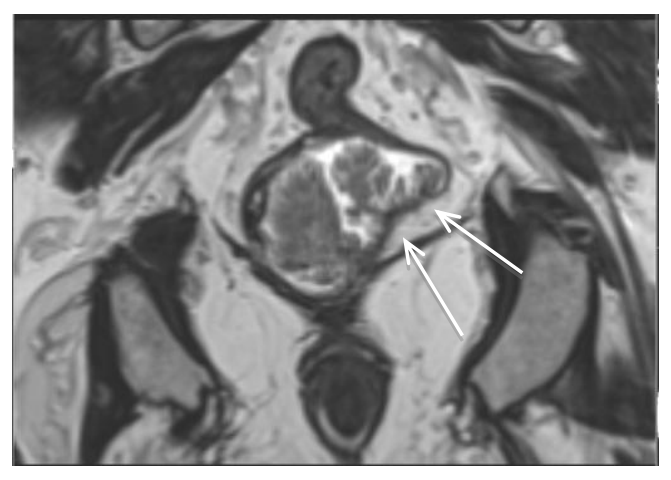

Fig. 4-58 year old patient with biopsy proven prostate carcinoma.

A: Oblique axial image demonstrating the T2 hypointense tumour involving the right apical and central gland (arrow), with no associated extra-capsular extension.

B: The carcinoma (arrow) displays low signal on the corresponding ADC map.

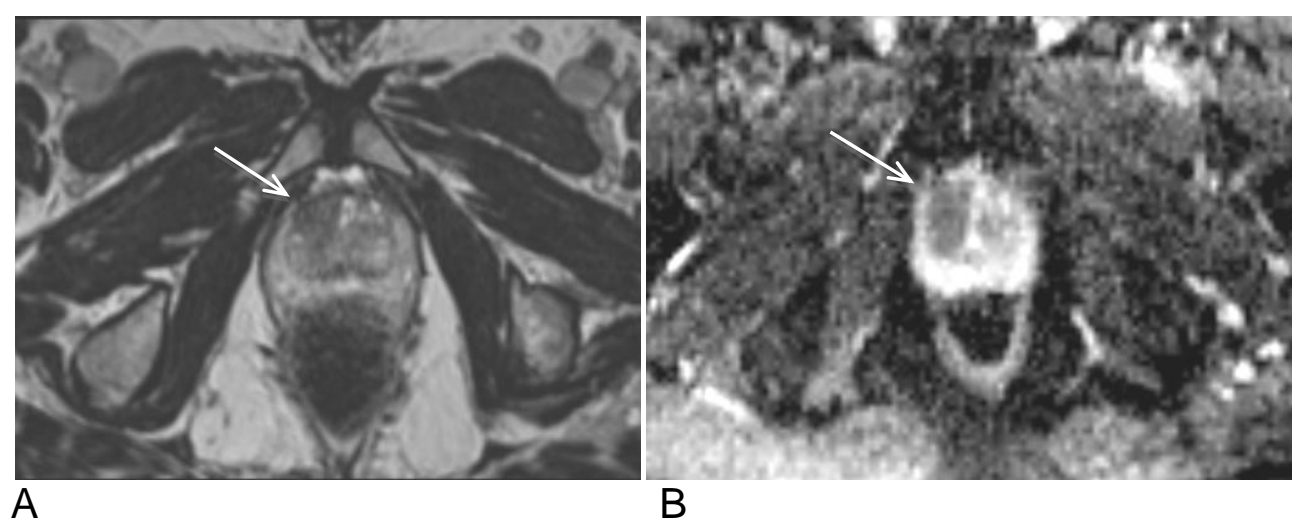


Fig. 5 - 28 year old male patient with known Crohn's disease with complex perianal fistulas.

A \& B: Sequential oblique axial images demonstrating the T2 hyperintense fistulous tracts. The internal origin of the fistula can be seen in image A, at the 5 to 6 o'clock position (white arrow). The fistulous tracts extend anteriorly bilaterally (dashed white arrows), posteriorly on the left (short open arrow) and towards and into the left ischioanal fossa (long open arrow).

C: Oblique coronal image demonstrating a fistulous tract extending superior to the levator ani consistent with a supralevator fistula (white arrow). On the right, a separate vertically-oriented fistulous tract can be traced to the cutaneous opening (black arrow). The multiplanar reformatting capability was extremely useful in this case for delineating the origin and course of each of the fistulous tracts.

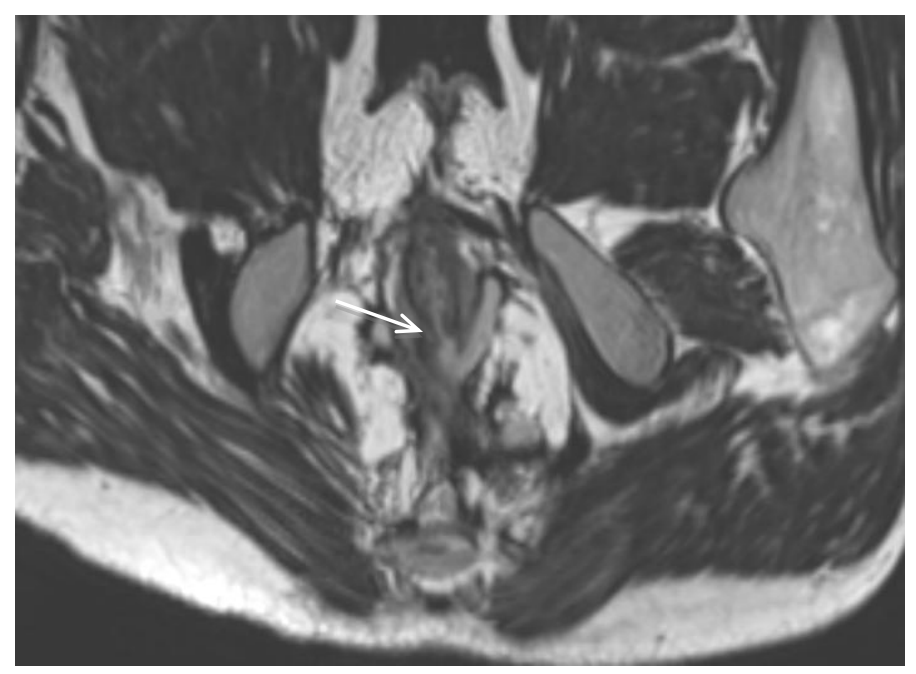

A

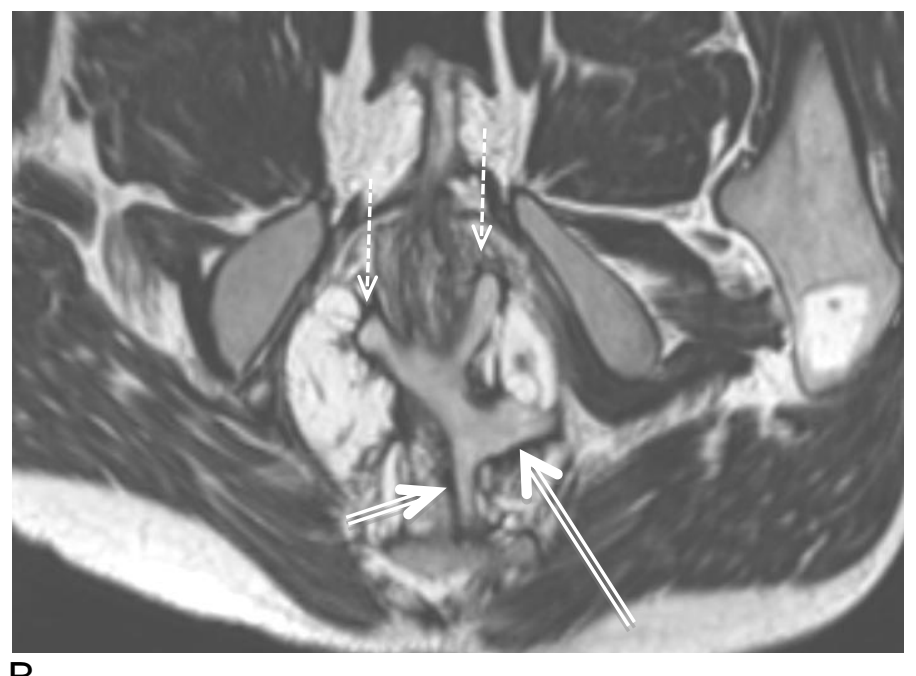

B 


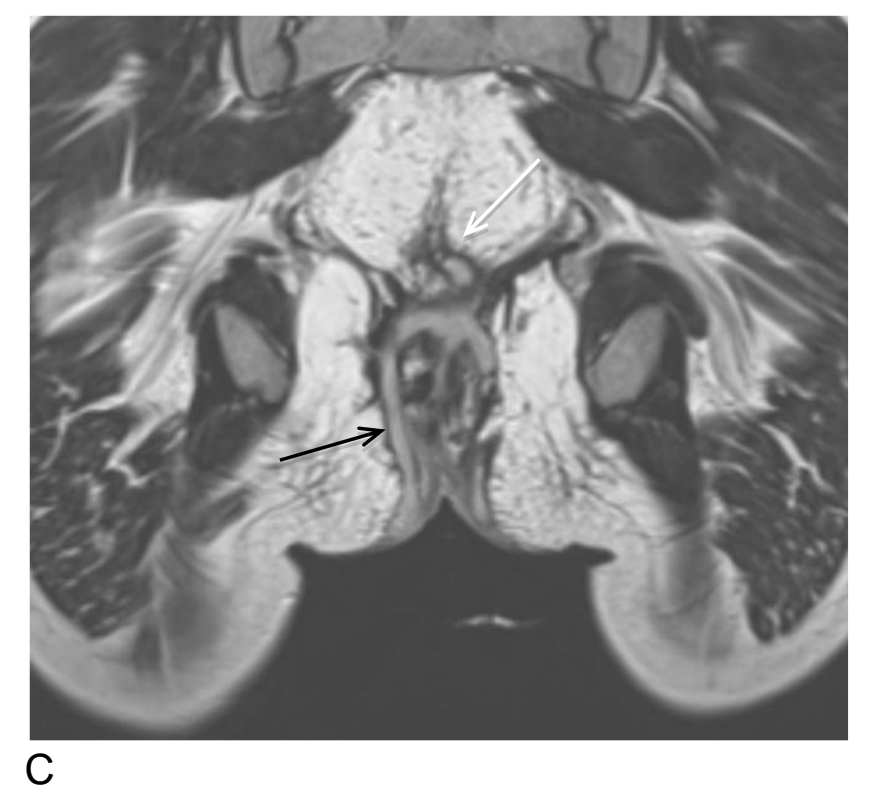


Fig 6 - Imaging appearance of endometriosis in several patients.

A: 29 year old patient with endometriosis. Axial reformats demonstrate Pouch of Douglas endometriotic deposit and bilateral ovarian endometriomas with indrawing of the ovaries (white arrows) towards the midline.

B: Same patient as in figure $6 \mathrm{~A}$. Axial reformats more inferiorly demonstrate tethering of the traversing rectum towards the left adnexal region (white arrow).

C: 34 year old patient with known severe endometriosis. Sagittal reformat demonstrating a large left sided ovarian endometrioma (white arrow) and endometriotic deposit (black arrow) in the uterine fundal region. There is tethering of the adjacent sigmoid to the posterior uterine body (dashed white arrow), without clear evidence of bowel wall invasion by endometriosis deposits.

D: 31 year old patient with a history of primary infertility. A diagnosis of endometriosis was made and the use of 3D T2 TSE has aided in the detection of other significant incidental findings. Axial reformat demonstrating extensive endometriosis in the Pouch of Douglas (white arrow). Incidental finding of two endometrial cavities.

$\mathrm{E}$ : Same patient as in figure 6D. The large FOV coronal reformat demonstrated mural thickening of small bowel loops more superiorly within the abdomen (white arrows) and the suspicion of Crohn's disease was raised. The patient was subsequently diagnosed with Crohn's disease on jejunal biopsy. This was a significant finding given some symptoms attributed to endometriosis were indeed symptomatic of Crohn's disease, the treatment of which is vastly different.

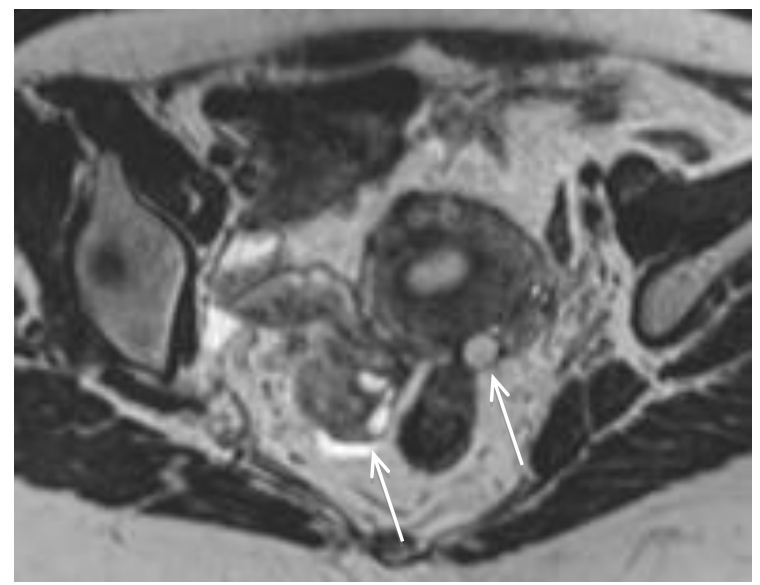

A 


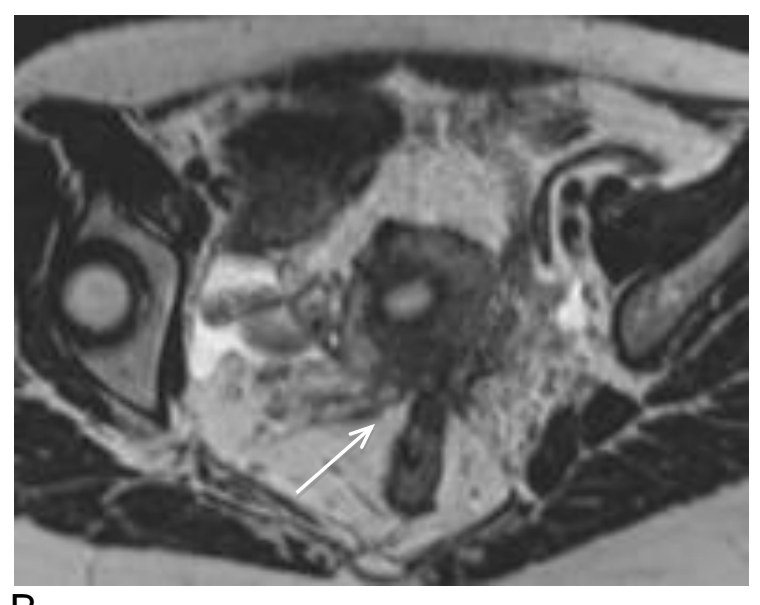

B
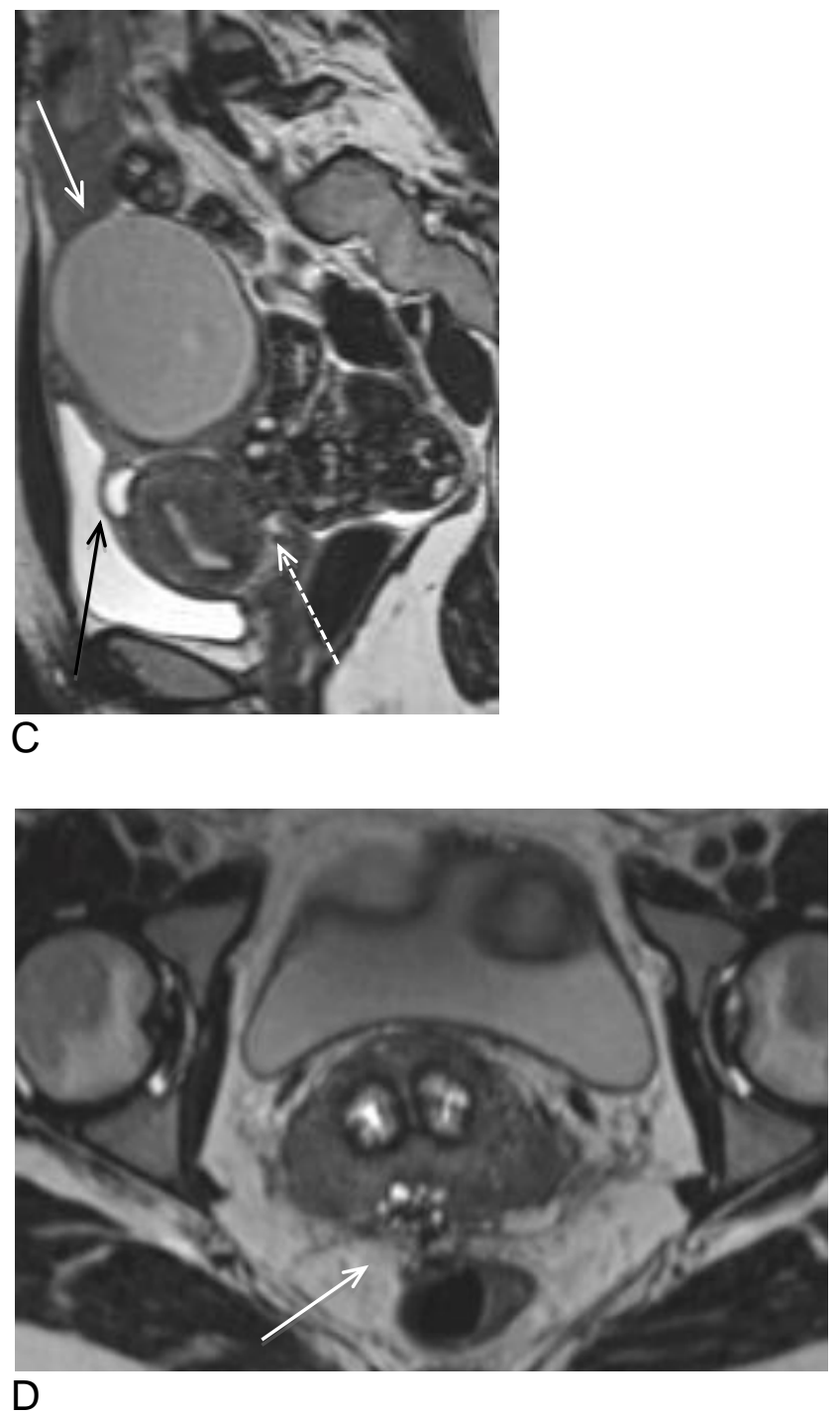


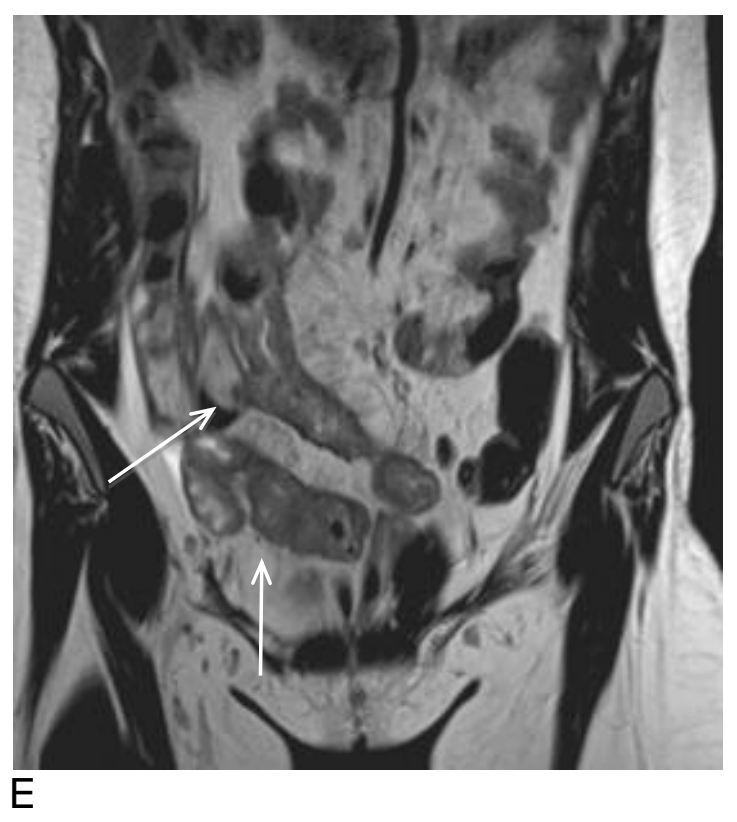


Fig. 7 - 74 year old patient with stage 2B cervical cancer.

A: Sagittal reformat demonstrates a focal cervical mass posteriorly, with associated disruption of the posterior low cervical stroma (arrow) and the likelihood of extension into the parametrial tissues was raised.

Fig b: On the axial imaging the parametrial extension was well displayed (arrow) in keeping with stage 2B disease. This was confirmed on postoperative histopathology.

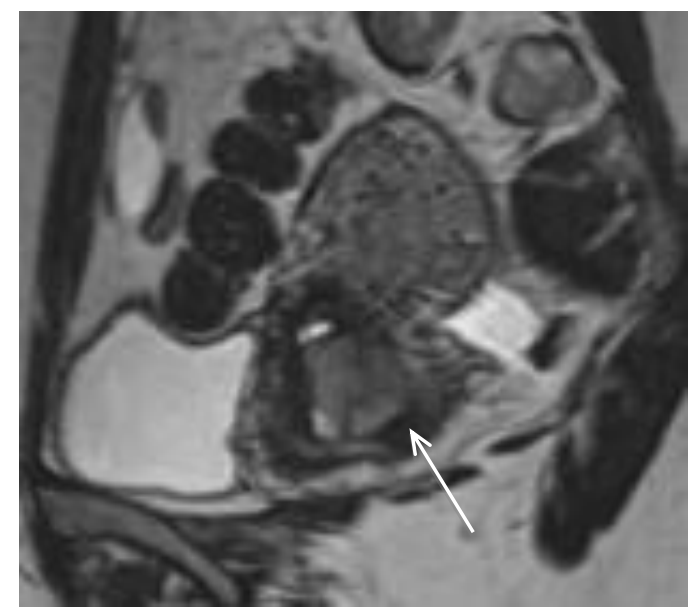

A

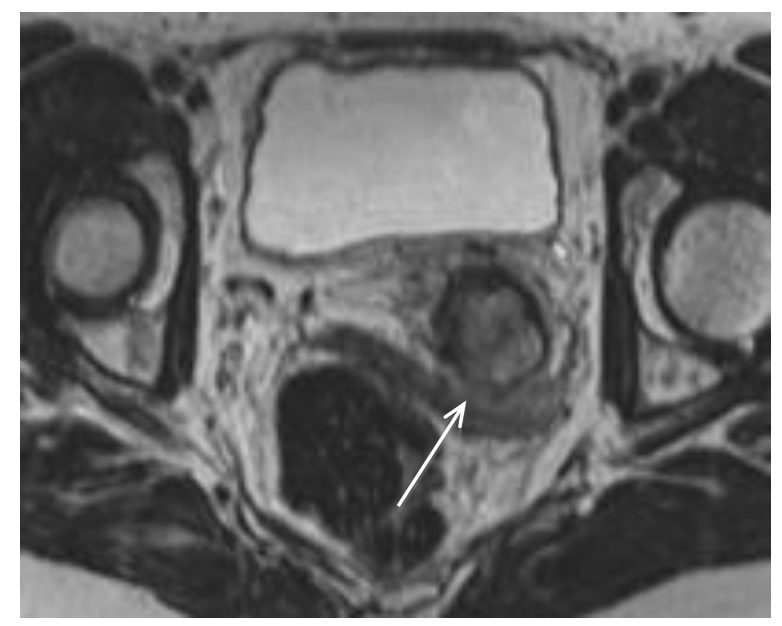

B 
Fig. 8 - 83 year old patient with endometrial carcinoma.

A: Irregular lobulated thickening of the endometrium arising from the left wall of the uterus towards the fundus with poor definition of the junctional zone.

There is invasion of more than half of the myometrium in keeping with a T1B tumour. Incidental finding of several uterine fibroids. Axial reformat demonstrating the close relationship between the uterine fibroid (white arrow) and the endometrial cancer (dashed white arrows).

B: Sagittal reformat shows that the tumour extends to and probably involves the fibroid in the fundal region of the uterus (arrow).

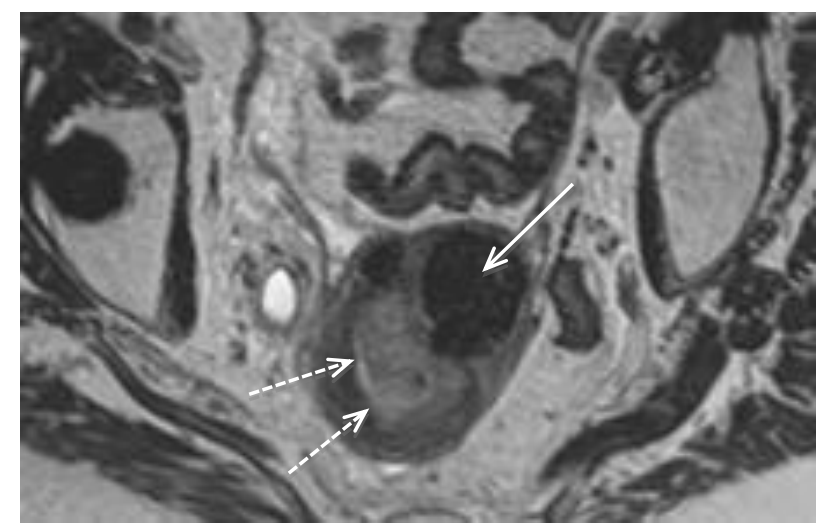

A

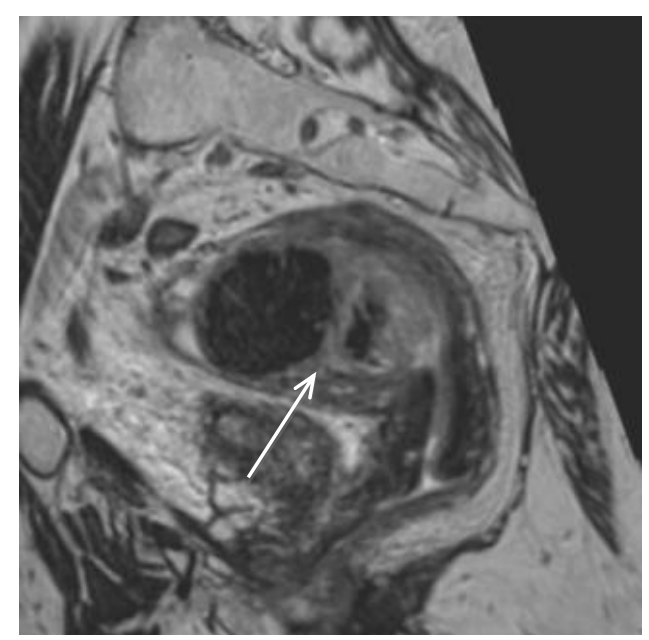

B 
Fig 9 - MRI appearance of congenital uterine anomalies in 2 patients.

A: 31 year old patent with uterus didelphys (same patient as in figure 6D \& $6 \mathrm{E})$. Coronal reformat demonstrating how 3DT2 MRI of the uterus can be helpful in identifying the type of uterine congenital anomaly as it allows us to reformat along the long axis and tilt/lie of the uterus. In this case we see complete duplication and separation of the uterine horns and cervices in keeping with uterus didelphys.

B: 31 year old patient with a suspected uterine anomaly for work-up. Oblique axial image demonstrating the slightly convex to flat fundal region of the uterus (arrow) in keeping with a septate uterus.

C: Same patient as in figure 9B. Oblique axial image more inferior to fig. 9B demonstrate a septum extending to the external cervical os in keeping with a complete septate uterus. The septum extends to the cervical os (arrow) on this axial reformatted image.

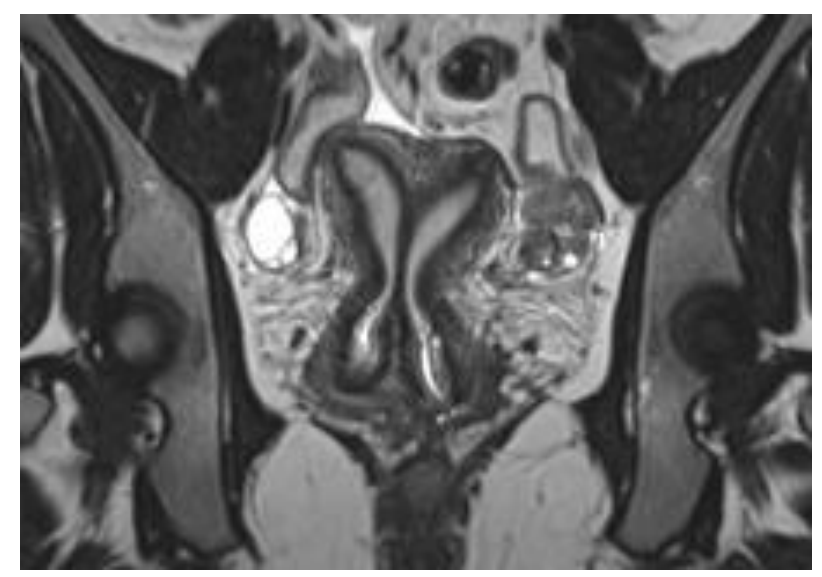

A

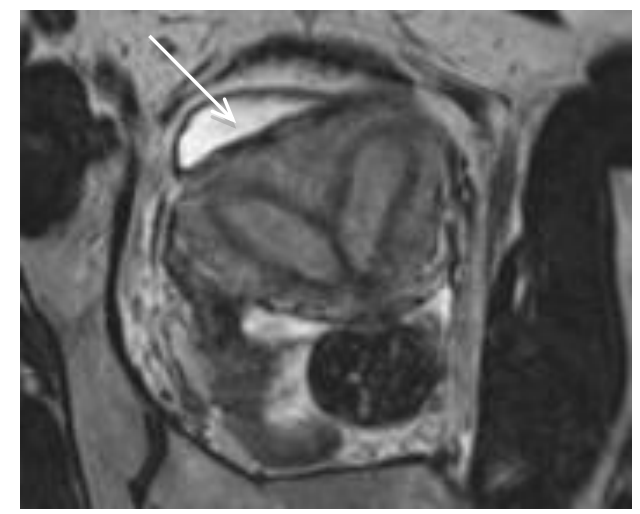

B 


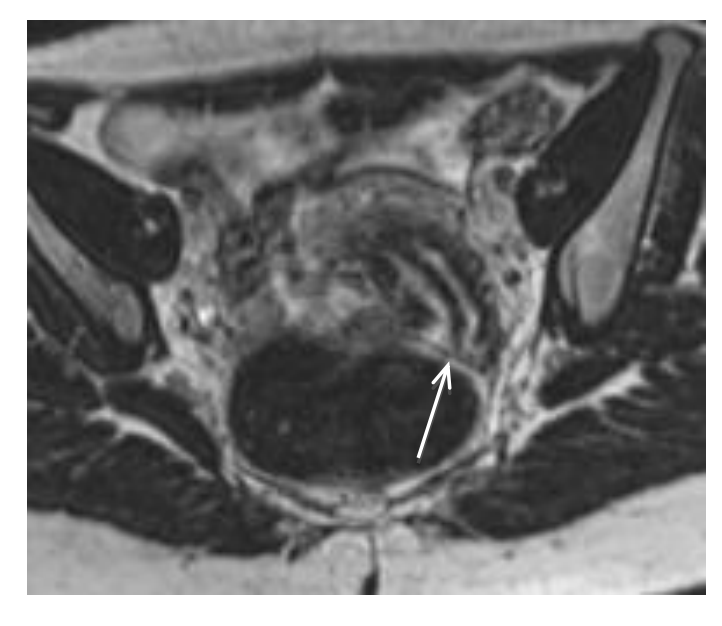

C 